\title{
Population Prediction Research in Zhejiang Province Based on BP Neural Network
}

\author{
Wanle Chi ${ }^{1, a^{*}}$ \\ ${ }^{1}$ Wenzhou Vocational \&Technical College, Zhejiang, 325000, China \\ a358455713@qq.com \\ * The Corresponding Author
}

Keywords: BP neural network; Prediction; Population

Abstract. The population growth problem has always been a key issue. So, a scientific and accurate forecast of the population is necessary. The population data are actually time series. The previous data has a non-linear relevance to the subsequent data. In this paper, a system model based on BP algorithm for forecast population growth in zhejiang province. This paper has collected the related population data in zhejiang province form statistics bureau of zhejiang province during the period of 1978 to 2016 (39 years). The simulation result was shown that the BP algorithm was effective and feasible in population prediction, and can achieve high accuracy.

\section{Introduction}

The population data often shows non-linear characteristics. Therefore, the traditional linear analysis method to forecast the future trend of the population growth has limitations, and it is difficult to get a satisfactory result. Artificial neural network has strong mapping ability of non-linear system. It can realize mapping relationship between $\mathrm{M}$ dimension vector to $\mathrm{N}$ dimension vector. And it doesn't need to use mathematical model of complex non-linear system. BP neural network is the abbreviation of erroneous reverse transmission neural network. BP neural network is presently one of the most successful and widely applied neural network models. The result of this paper shows that the BP neural network is effective and feasible in population forecast, and can achieve high accuracy, and has certain practical value.

\section{Algorithm \& Data}

The Construction of Neural Network. BP neural network generally has three layers of network structure, including input layer, hidden layer and output layer. The neurons of each layer are connected only with the adjacent layer neurons. But there is no connection between the neurons at the same layer. The adjacent layer neurons are connected by the weight value. The structure of BP neural network is shown in Fig 1.

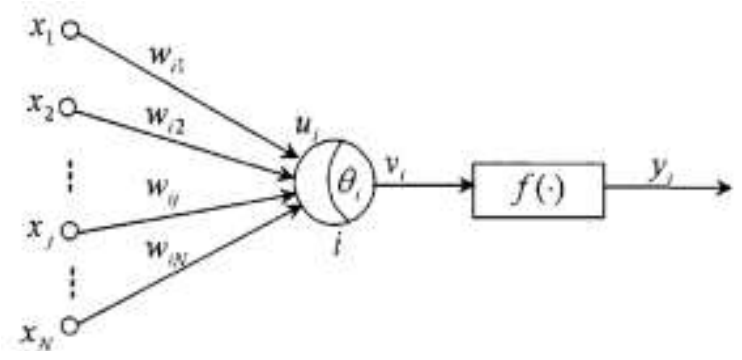

Figure 1. The structure of three layers BP neural network

The BP algorithm has the forward propagation signal and the reverse propagation error. In the forward propagation, the input data are propagated from the input layer to the hidden layer. After the excitation function, the data of the hidden layer is transmitted to the output layer. The output result is obtained by the excitation function of the output layer.

In the forward learning process, the weights of each node on the input layer, the hidden layer and the output level are random allocation values. If there is oversize error between the error of the output 
value and the real value, the processing results are retained and entered into the reverse phase of the error. The error is reduced by the error gradient through the output layer by modifying the weights of each layer. The error is corrected by the error gradient in the output layer, and the weights are corrected in the way of error gradient. The back propagation is hidden back to the hidden layer and input layer. So the output of the network keeps approaching the desired output until the operation result reaches satisfactory accuracy or preset learning step ${ }^{[1,2]}$.

Data. This paper has collected the related population data in zhejiang province form statistics bureau of zhejiang province during the period of 1978 to 2016 (39 years), and the first 26 years as a training set, the last 13 years as a test set.

These are the input data $\mathrm{p}$, including total population, GDP, per capita consumption, birth population and death population. The total population of the next year is as the output data t. Some data are as Table 1.

Table 1 Some of original data

\begin{tabular}{ccccccc}
\hline Year & $\begin{array}{c}\text { Total } \\
\text { population }\end{array}$ & GDP & $\begin{array}{c}\text { Per } \\
\text { consumption }\end{array}$ & $\begin{array}{c}\text { Birth } \\
\text { population }\end{array}$ & $\begin{array}{c}\text { Death } \\
\text { population }\end{array}$ & $\begin{array}{c}\text { Next total } \\
\text { population }\end{array}$ \\
\hline 1978 & 3750.96 & 123.72 & 193.00 & 67.75 & 21.75 & 3792.33 \\
\hline 1979 & 3792.33 & 157.75 & 218.00 & 67.82 & 22.23 & 3826.58 \\
\hline 1980 & 3826.58 & 179.92 & 240.00 & 59.40 & 23.97 & 3871.51 \\
\hline 1981 & 3871.51 & 204.86 & 317.00 & 69.00 & 24.12 & 3924.32 \\
\hline
\end{tabular}

It's necessary to normalize the input of sample in order to calculate conveniently and prevent partial neurons to supersaturate.

$$
P=\frac{p-p_{\min }}{p_{\max }-p_{\min }}
$$

In formula 1 , the $\mathrm{p}$ is the input matrix. $\mathrm{P}$ is the row normalized matrix. And the output vector $\mathrm{t}$ is normalized as follow formula $2^{[3]}$ :

$$
\mathrm{T}=\frac{\mathrm{t}-\mathrm{t}_{\min }}{\mathrm{t}_{\max }-\mathrm{t}_{\min }}
$$

\section{Train}

The Transfer Function and Training Function. In this paper, a three layers BP neural network prediction system with a hidden layer is used to predict the population. The hidden nodes are determined by heuristics algorithm, and the number of output nodes is 1 .

The transfer function choose purelin or tansig. The log - s - function has better fitting effect, so the tansig function is selected to connect the input layer and the hidden layer. The transfer function between the hidden layer and the output layer is the purelin function. Because the input and output values are normalized in $[0,1]$ interval, this is in line with the actual comparison ${ }^{[4,5]}$.

The model of BP neural network has many training functions, such as traingd, traingdm, traingda, traincgf, traincgp, traincgb, trainscg, trainbfg, trainbr, trainlm. MAPE (mean absolute percentage error) is used to test the fitting result of train. The heuristics algorithm is used to determine the number of neurons in hidden layer. The number of hidden layer neurons is tentative designed to 6 16 . By testing with different number neurons and different training functions, the results are shown in Table 2. 
Table 2 The training function test \& MAPE value

\begin{tabular}{|c|c|c|c|c|c|c|c|c|c|c|c|}
\hline \multirow{2}{*}{$\begin{array}{r}\text { Trainin } \\
\mathrm{g} \\
\text { Functio } \\
\mathrm{n}\end{array}$} & \multicolumn{11}{|c|}{ MAPE(with different number neurons in ) } \\
\hline & 6 & 7 & 8 & 9 & 10 & 11 & 12 & 13 & 14 & 15 & 16 \\
\hline \multirow{2}{*}{ traingd } & 3.395 & 2.105 & 1.285 & 1.903 & 1.980 & 1.355 & 1.696 & 1.813 & 1.942 & 1.999 & 2.010 \\
\hline & 4 & 8 & 4 & 7 & 7 & 3 & 2 & 1 & 2 & 4 & 4 \\
\hline traingd & 3.477 & 1.405 & \multirow{2}{*}{2.074} & 1.837 & 1.898 & 1.491 & 2.074 & 1.473 & 1.305 & 1.558 & 1.295 \\
\hline $\mathrm{m}$ & 2 & 9 & & 5 & 7 & 7 & 3 & 8 & 3 & 5 & 6 \\
\hline traingd & 1.161 & 0.854 & 0.998 & 0.848 & 1.261 & 18 & 0.773 & 1.508 & 1.599 & 1.613 & 1.341 \\
\hline $\mathrm{a}$ & 2 & 1 & 2 & 7 & 8 & 4 & 4 & 6 & 3 & 6 & 5 \\
\hline \multirow{2}{*}{ traincgf } & 5.134 & 0.557 & 0.714 & 0.571 & 0.488 & 0.502 & 0.462 & 0.554 & 0.480 & 0.612 & 0.534 \\
\hline & 3 & 6 & 7 & 7 & 9 & 1 & 9 & 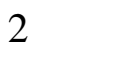 & 6 & 2 & 6 \\
\hline traincg & 1.370 & 0.846 & 0.626 & 0.845 & 0.453 & 0.608 & 0.479 & \multirow{2}{*}{0.425} & 0.553 & 0.345 & 0.567 \\
\hline $\mathrm{p}$ & 2 & 6 & 6 & 8 & 2 & 1 & 6 & & 6 & 3 & 2 \\
\hline \multirow{2}{*}{$\begin{array}{l}\text { traincg } \\
\mathrm{b}\end{array}$} & 0.464 & \multirow{2}{*}{0.465} & 0.397 & 1.580 & 0.688 & 0.733 & 0.286 & 0.701 & 0.725 & 0.673 & 0.286 \\
\hline & 3 & & 4 & 2 & 3 & 8 & 9 & 1 & 1 & 4 & 3 \\
\hline \multirow{2}{*}{ trainscg } & 0.161 & \multirow{2}{*}{0.151} & \multirow{2}{*}{0.112} & 0.111 & 0.091 & 0.075 & 0.082 & 0.097 & 0.101 & 0.115 & 0.080 \\
\hline & 4 & & & 5 & 7 & 1 & 8 & 1 & 7 & 4 & 2 \\
\hline \multirow{2}{*}{ trainbfg } & 0.300 & 0.130 & 0.101 & 0.123 & 0.120 & 0.099 & 0.104 & 0.109 & 0.107 & 0.090 & 0.106 \\
\hline & 7 & 4 & 5 & 8 & 2 & 1 & 2 & 3 & 3 & 6 & 5 \\
\hline \multirow{2}{*}{ trainbr } & 0.562 & 0.562 & 0.562 & 0.562 & 0.562 & 0.562 & 0.585 & \multirow{2}{*}{0.585} & \multirow{2}{*}{0.585} & 0.584 & 0.562 \\
\hline & 3 & 4 & 4 & 5 & 5 & & & & & & \\
\hline \multirow{2}{*}{ trainlm } & 0.129 & 0.121 & 0.060 & 0.097 & 0.122 & 0.064 & $\underline{0.039}$ & 0.109 & 0.097 & 0.075 & 0.106 \\
\hline & 7 & 3 & 7 & 9 & 2 & 3 & $\underline{3}$ & 9 & & & 4 \\
\hline
\end{tabular}

Based on the same transfer function, Table 2 is obtained by changing the training function on the premise of target error 0.000001 . According to the minimum MAPE criterion, the training function of trainlm is selected.

The Number of Neurons in the Hidden Layer. The heuristics algorithm is use to determine the final number of neurons in hidden layer too. The number of hidden layer neurons is tested to 2 26. The best number is selected with the minimum MAPE criterion. The maximum iterations number is 10000. And the goal error is 0.0000001 . The learning rate is set to 0.05 . The momentum factor is 0.9 . The results are shown as Table 3 .

Table 3 The number of neurons \& MAPE value

\begin{tabular}{|c|c|c|c|c|c|c|c|}
\hline \multirow{3}{*}{$\begin{array}{l}\text { neuron number } \\
\text { MAPE value }\end{array}$} & 2 & 3 & 4 & 5 & 6 & 7 & 8 \\
\hline & 0.473 & 0.141 & 0.121 & 0.123 & \multirow{2}{*}{0.091} & 0.122 & 0.075 \\
\hline & 9 & 3 & 3 & 9 & & 6 & 3 \\
\hline \multirow{3}{*}{$\begin{array}{l}\text { neuron number } \\
\text { MAPE value }\end{array}$} & 9 & 10 & 11 & 12 & 13 & 14 & 15 \\
\hline & 0.095 & 0.112 & 0.070 & 0.114 & \multirow{2}{*}{0.083} & 0.005 & 0.012 \\
\hline & 3 & 6 & 8 & 7 & & 9 & 5 \\
\hline \multirow{3}{*}{$\begin{array}{l}\text { neuron number } \\
\text { MAPE value }\end{array}$} & 16 & 17 & 18 & 19 & 20 & 21 & 22 \\
\hline & 0.077 & 0.058 & 0.021 & 0.122 & 0.001 & 0.013 & 0.003 \\
\hline & 5 & 4 & 5 & 9 & $\underline{5}$ & 1 & 4 \\
\hline \multirow{3}{*}{$\begin{array}{l}\text { neuron number } \\
\text { MAPE value }\end{array}$} & 23 & 24 & 25 & 26 & & & \\
\hline & 0.072 & 0.032 & 0.003 & 0.003 & & & \\
\hline & 6 & 5 & 3 & 4 & & & \\
\hline
\end{tabular}

The result is shown the MAPE value of 20 neurons is lest. The final model of BP neural network is the model of 20 neurons in hidden layer with the trainlm training function.

Result. The results of train are follows: 


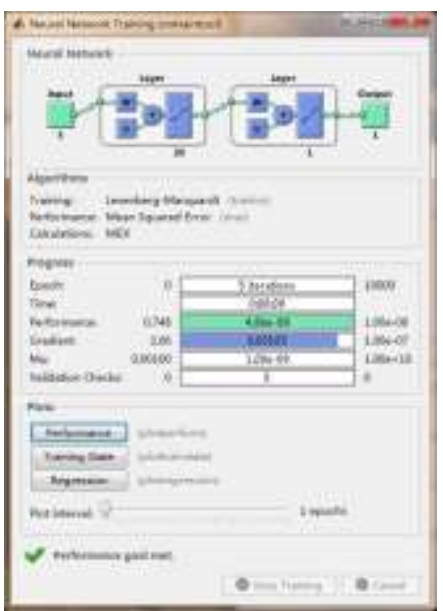

Figure 2. The graph of training

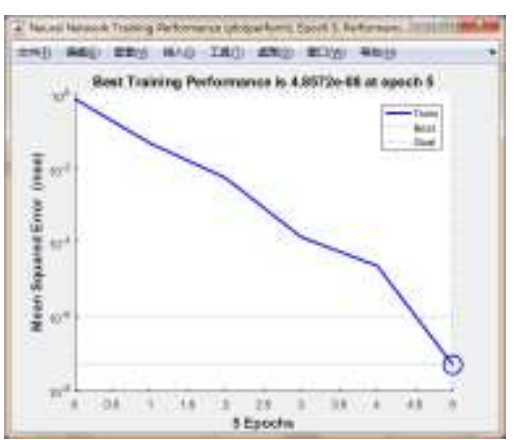

Figure 3. The performance

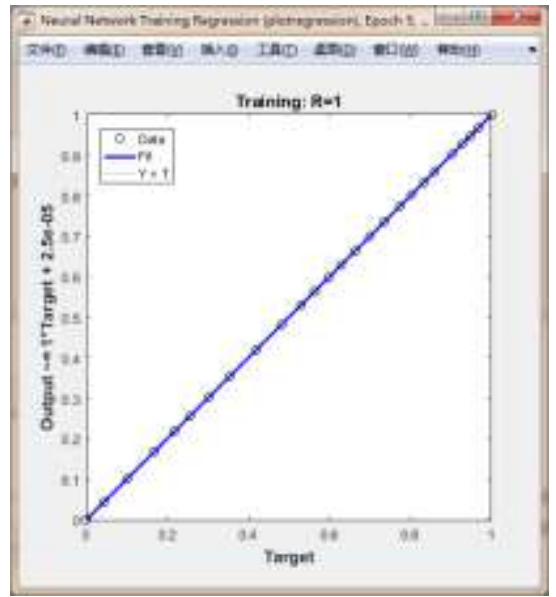

Figure 4. The regression

From Fig 2 and Fig 3 , the number of iterations is 5 .It shows that the training gradient descends quickly. The computational performance is excellent. From Fig 4 , the fitting result is closer to the real value.

\section{The Simulation of BP Neural Network}

Test Data. Some of test data are shown as Table 4. The test data are total population during the period of 2004 to 2015 (12 years).

Table 4 Some of test data

\begin{tabular}{ccccccc}
\hline Year & $\begin{array}{c}\text { Total } \\
\text { population }\end{array}$ & GDP & $\begin{array}{c}\text { Per } \\
\text { consumption }\end{array}$ & $\begin{array}{c}\text { Birth } \\
\text { population }\end{array}$ & $\begin{array}{c}\text { Death } \\
\text { population }\end{array}$ & $\begin{array}{c}\text { Next total } \\
\text { population }\end{array}$ \\
\hline 2004 & 4577.22 & 11648.70 & 8174.00 & 50.12 & 26.95 & 4602.11 \\
\hline 2005 & 4602.11 & 13417.68 & 9558.00 & 54.37 & 29.78 & 4629.43 \\
\hline 2006 & 4629.43 & 15718.47 & 11099.00 & 50.78 & 26.75 & 4659.34 \\
\hline 2007 & 4659.34 & 18753.73 & 12730.00 & 52.11 & 27.96 & 4687.85 \\
\hline
\end{tabular}

Results. As shown in Table 5, the prediction results of numerical simulation show a good agreement with the real values. The MAPE of forecasting is $0.28 \%$.

Table 5 Result of forecasting

\begin{tabular}{ccccc}
\hline Year & Real data & Simulation data & Difference & APE \\
\hline 2004 & 4602.11 & 4617.025 & -14.91 & 0.324088 \\
\hline 2005 & 4629.43 & 4613.002 & 16.43 & 0.354867 \\
\hline 2006 & 4659.34 & 4684.401 & -25.06 & 0.537876 \\
\hline 2007 & 4687.85 & 4675.394 & 12.46 & 0.265715 \\
\hline 2008 & 4716.18 & 4722.248 & -6.07 & 0.128662 \\
\hline 2009 & 4747.95 & 4734.473 & 13.48 & 0.283843 \\
\hline 2010 & 4781.31 & 4810.777 & -29.47 & 0.616303 \\
\hline 2011 & 4799.34 & 4800.951 & -1.61 & 0.033563 \\
\hline 2012 & 4826.89 & 4824.969 & 1.92 & 0.03979 \\
\hline 2013 & 4859.18 & 4869.467 & -10.29 & 0.211698 \\
\hline 2014 & 4873.34 & 4889.36 & -16.02 & 0.328729 \\
\hline
\end{tabular}




\section{Summary}

Using the total population of zhejiang, the BP neural network is applied to population prediction. And the results show that the BP neural model has accuracy to forecast the population growth.

\section{Acknowledgments}

This work was supported by Wenzhou vocational \& technical college scientific research project fund(wzy2016019).

\section{References}

[1] Jian Song, Jingyuan Yu, Guangyuan Li, Forecast population development process , Science China Press.9 (1980) 920-933.

[2] Chunhua Yin, Li Chen, The Research and application of population prediction based on BP neural network model, Population Journa1.2 (2005)44-48.

[3] Xin Yao, Yong Liu. A new evolutionary system for evolving artificial neural networks, Transaction on Neural Networks. 8 (2007) 34-37.

[4] Jin Xu. A new algorithm for feed forward neural network learning and its simulation, Journal of Commercial University: Natural Science Edition. 4 (2007) 1-3.

[5] Yuxi Wang, Hong Wang, Forecast on population distribution of shanghai pension system based on the gray model. Systems Engineering Theory\& Practice.30(2010)2244-2253. 